\title{
HARMONIZED DECISION-MAKING IN MANAGING RELIABILITY AND SAFETY
}

\author{
Avner Ben-Yair ${ }^{1}$, Dimitri Golenko-Ginzburg ${ }^{2}$, Zohar Laslo ${ }^{3}$ \\ ${ }^{1}$ Department of Industrial Engineering and Management, Sami Shamoon College of Engineering, Beer-Sheva, 84100, \\ Israel, E-mail: avnerb@sce.ac.il \\ ${ }^{2}$ Department of Industrial Engineering and Management, Ben-Gurion University of the Negev, Beer-Sheva, 84105, \\ Israel,E-mail: dimitri@bgumail.bgu.ac.il \\ Department of Industrial Engineering and Management, Academic College of Judea and Samaria, Ariel, 44837 \\ ${ }^{3}$ Department of Industrial Engineering and Management, Sami Shamoon College of Engineering, Beer-Sheva, 84100, \\ Israel,E-mail: zohar@sce.ac.il
}

Received 0601 2005; accepted 10022005

\begin{abstract}
A hierarchical technical system functioning under random disturbances and being subject to critical failures at the bottom level which may result in an accident or a hazardous condition including environmental safety violations at the upper level is considered. Certain primary elements at the bottom level, together with their corresponding primary failures, can be refined by undertaking technical improvement. The list of the latter is pre-given as well. Assume that by means of simulation modeling (SM) it is possible to evaluate the increment of the system's reliability by implementing any set of technical improvements. The harmonization models center on determining an optimal sub-set of technical improvements in order:

- either to maximize the system's reliability subject to a restricted budget assigned for the improvements' implementation, or

- to minimize the system's budget subject to a reliability value restricted from below.
\end{abstract}

Keywords: Harmonization models; Basic parameters; Multi-parametric trade-off models; Hierarchical safety engineering models; Heuristic models based on sensitivity concepts.

\section{Introduction}

In recent years problems associated with developing various quality concepts have been discussed extensively in scientific literature. However, numerous publications refer mostly to quality control which is usually applied to products and services. As a matter of fact, the existing quality techniques, including the developed utility theory $[7,13]$, are not applicable to technical and organization systems which are actually supervising and monitoring the process of the system functioning: all those models are restricted to solving market competitive problems alone. Thus, nowadays, the existing utility theory centers on analyzing the competitive quality of organization systems' outcome products rather than dealing with the quality of the systems' functioning, i.e., with organization systems in their entirety. This may result in heavy financial losses, e.g. when excellent project objectives are achieved by a badly organized project's realization [4].

Thus, a conclusion can be drawn that the existing utility theory cannot be used as the system's quality techniques. In order to fill in the gap, we have undertaken research in the area of estimating the quality of the system itself, e.g. the system's public utility. We will consider complex organization system which functions under random disturbances. Such a system usually comprises a variety of qualitative and quantitative attributes, characteristics and parameters, which enable the system's functioning. The problem arises to determine a generalized (usually quantitative) value which covers all essential system's parameters and can be regarded to as a system's qualitative estimate. We will henceforth call such a generalized value the system's utility.

Another conclusion which can be drawn from the outlined above reference is that the creators of the existing utility theory have implemented safety concepts only in techniques to control the outcome products. Any research to estimate the system's utility as a whole from the point of Safety Engineering or Environmental Safety principles has not been published as yet [11]. Since considering safety concepts in modern operation management becomes a growing world-wide tendency, we will implement those concepts in the utility of the system's functioning. This, in turn, will result in decreasing the number of hazardous failures jeopardizing public and environmental safety in modern organization systems [2]. 
In papers $[1,2,8]$ we have created a new multiparametric optimization model in order to maximize the system's utility as a generalized quality measure of the system's functioning. Since such a model is, in essence, a trade-off compromise between the system's parameters, we will henceforth call that model harmonization model (HM).

We suggest calling the system's utility a weighted linear function of the system's parameters with constant coefficients. The parameters are divided into:

- independent parameters, where for each parameter its value may be preset and may vary independently on other parameters' values, and - dependent parameters whose values may not depend uniquely on the values of independent parameters. However, when optimized (for the same values of independent parameters), they are solely dependent on those values.

Both independent and dependent parameters together with the coefficients of the utility function are externally pre-given.

If an organization system functions under random disturbances and comprises $n_{1}$ independent basic parameters $R_{i}^{(i n d)}, 1 \leq i \leq n_{1}$, and $n_{2}$ dependent basic parameters $R_{j}^{(d e p)}, 1 \leq j \leq n_{2}$, the harmonization problem boils down to maximize the system's utility

$$
U_{S}=\left(\sum_{i=1}^{n_{1}} \alpha_{i} R_{i}^{(i n d)}+\sum_{j=1}^{n_{2}} \alpha_{j} R_{j}^{(d e p)}\right)
$$

subject to certain restrictions. We suggest determining the optimal vector

$$
\vec{R}_{*}=\left(R_{1 *}^{(i n d)}, R_{2 *}^{(i n d)}, \ldots, R_{n_{1} *}^{(i n d)}, R_{1 *}^{(d e p)}, R_{2 *}^{(d e p)}, \ldots, R_{n_{2} *}^{(d e p)}\right)(2)
$$

which delivers maximization to the system's utility $U_{S}$, by means of the following sequential stages:

Stage I - implement a look-over algorithm to examine all feasible combinations of independent basic values $\left\{R_{i}{ }^{(\text {ind })}\right\}$;

Stage II - determine optimal values $\left\{R\left(\begin{array}{l}\text { (dep } \\ j \text { opt }\end{array}\right)\right\}$ for all dependent parameters by means of values $\left\{R_{i}^{(\text {ind })}\right\}$ obtained at the previous stage; for each $j$-th dependent parameter an individual optimization model (called henceforth the partial harmonization model $\left.P H M_{j}\right)$, is used. The latter enables the optimality of each value $R_{j \text { opt }}^{(\text {dep })}$ which solely depends on the combination $\left\{R_{i}^{(\text {ind })}\right\}$;

Stage III- calculate the system's utility $U_{S}$ via (1) for the combination

$$
\left\{R_{i}^{(\text {ind })}\right\},\left\{R_{j \text { opt }}^{(\text {dep })}\right\}
$$

obtained at Stages I and II;

Stage IV- Calculate the optimal system's utility by determining the optimal combination (2) for all independent and dependent parameters which delivers the maximum to $U_{s}$.

If, due to the high number of possible combinations $\left\{R_{i}^{(i n d)}\right\}$, implementing Stage I requires a lot of computational time, we suggest using a simplified heuristic search procedure, e.g. a cyclic coordinate search algorithm [1,8].

Thus, we suggest an approximate harmonization's problem solution as follows. At the first stage a relatively simple search algorithm in the area of independent parameters is implemented. At the second stage, in order to evaluate the optimal value of each dependent parameter, an optimization problem

$\operatorname{PHM}_{j}, 1 \leq j \leq n_{2}$, has to be solved. Thus, the idea is to obtain independent parameters' values at the first stage and to use them as input values of all partial harmonization models at the second stage.

PHM is usually a stochastic optimization model which is solved on the basis of simulation modeling. However, in certain cases, e.g. reliability and safety engineering problems, various $P H M$ require more complicated formulations. In such cases we suggest to use additional heuristic models in order to implement realistic quantitative links between the system's attributes. For various dependent parameters the $P H M$ may be formulated and solved by means of expert information $[1,8]$.

The goal of this paper is to apply the harmonization theory in Reliability and Safety Engineering. A hierarchical technical system functioning under random disturbances and being subject to critical failures at the bottom level which may result in an accident or a hazardous condition including environmental safety violations at the upper level is considered. Certain primary elements at the bottom level, together with their corresponding primary failures, can be refined by undertaking technical improvement. The list of the latter is pre-given as well. Assume that by means of simulation modeling (SM) it is possible to evaluate the increment of the system's 
reliability by implementing any set of technical improvements $[1,3]$. The harmonization models center on determining an optimal sub-set of technical improvements in order:

- either to maximize the system's reliability subject to a restricted budget assigned for the improvements' implementation, or

- to minimize the system's budget subject to reliability value restricted from below.

Two different cases are considered and solved by mean of sensitivity analysis:

- a simplified cost-sensitivity trade-off model to solve cost-reliability problems for a complicated hazardous technical system with two basic parameters: cost and reliability, and

- more complicated trade-off harmonization problems where the system's utility, cost expenditures, reliability values and other basic parameters are linked together by means of sensitivity relations.

\section{Cost-reliability models}

\subsection{The System's Description}

We will consider a complicated technical device functioning under random disturbances. The device's reliability, i.e., its probability to avoid critical failures within a sufficiently long period of time, has to be extremely high since critical failures present a definite threat to people's safety, to the environment, etc., and may result in an accident or a major hazardous condition. Thus, increasing the device's reliability is considered to be an important problem of Safety Engineering, on assumption that the existing reliability value proves to be insufficient $[2,15]$.

Consider, further, that there exist $N$ technical improvements $(T I)$ to increase the device's reliability. For each $k$-th $T I, 1 \leq k \leq N$, investing $\Delta C_{k}$ cost expenditures results in increasing the device's reliability by $\Delta R_{k}$. Assume that those parameters are obtained by means of simulation model $S M$ and do not depend on the number of technical improvements which have already been implemented. Thus, the result of a routine $k$-th technical improvement does not depend on other $\{T I\}$.

The problems to be considered below present simplified particular cases of the general theory of harmonization models outlined in [1]. However, an effective and simple heuristic approach based on costsensitivity, can be suggested. To our opinion, the developed models can be applied to a broad spectrum of technical devices in the framework of Safety Engineering [1, 10].

\subsection{Notation}

Let us introduce the following terms:

\subsubsection{Cost-Reliability Models in Safety Engineering}

\begin{tabular}{|c|c|}
\hline$T I_{k}$ & $\begin{array}{l}\text { - the } k \text {-th technical improvement to } \\
\text { increase the system's reliability, }\end{array}$ \\
\hline$N$ & $\begin{array}{l}\text { - the number of possible technical } \\
\text { improvements; }\end{array}$ \\
\hline$\Delta C_{k}$ & $\begin{array}{l}\text { cost expenditures to implement } T I_{k} \\
\text { (pre-given); }\end{array}$ \\
\hline$\Delta R_{k}$ & $\begin{array}{l}\text { - increase of the system's reliability due } \\
\text { to implementing } T I_{k} \text { (to be } \\
\text { calculated by means of simulation } \\
\text { model } S M \text { ); }\end{array}$ \\
\hline$R^{*}$ & $\begin{array}{l}\text { - the minimal acceptable system's } \\
\text { reliability value to avoid hazardous } \\
\text { failures (pre-given); }\end{array}$ \\
\hline$C^{*}$ & $\begin{array}{l}\text { the restricted budget to undertake } \\
\text { technical improvements (pre-given); }\end{array}$ \\
\hline$R_{0}$ & $\begin{array}{l}\text { system's reliability value prior to } \\
\text { undertaking amendments (pre-given); }\end{array}$ \\
\hline$S M$ & $\begin{array}{l}\text { - simulation model to estimate the } \\
\text { system's reliability. }\end{array}$ \\
\hline
\end{tabular}

\subsubsection{Harmonization Models in Safety Engineering}

TS - Complicated multi-level technical system with hazardous failures at the upper level;

$N \quad$ the number of possible technical

improvements $T I_{k}, 1 \leq k \leq N$;

$\Delta C_{k} \quad$ - cost value required to carry out $T I_{k}$;

$S M \quad$ - simulation model to calculate the system's reliability value;

$\Delta R_{k} \quad-\quad$ additional reliability value obtained as a result of undertaking $T I_{k}$ (to be calculated by means of simulation model $S M$ );

$P_{\ell} \quad$ - additional non-basic parameter, $1 \leq \ell \leq m$;

$m$ - the number of non-basic parameters;

$\alpha_{C} \quad-\quad$ the budget's partial utility;

$\alpha_{R} \quad$ - the reliability's partial utility;

$\alpha_{P_{\ell}} \quad-\quad$ partial utility of parameter $P_{\ell}$;

$R^{*} \quad$ - the system's reliability level to avoid hazardous failures (pre-given);

$C^{*} \quad$ - maximal additional budget (pregiven); 


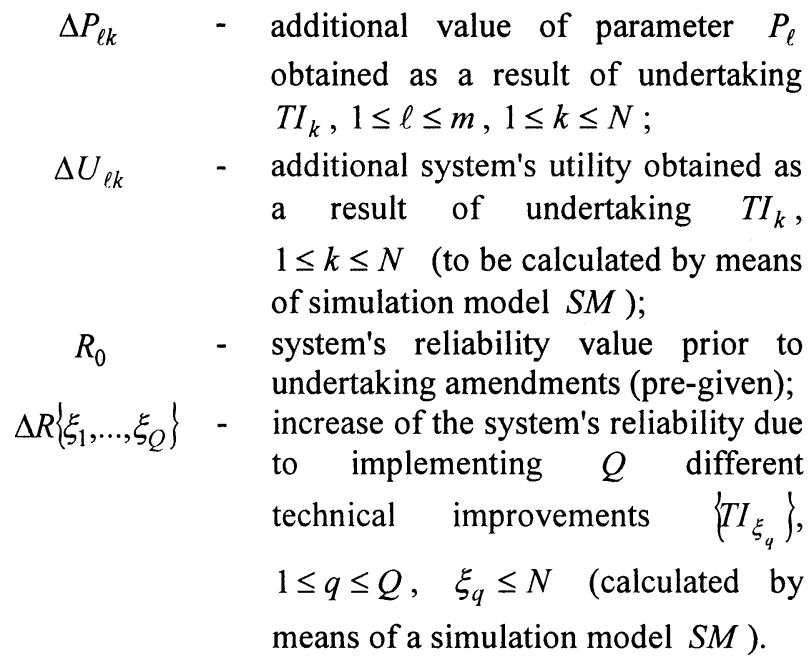

\subsection{The Direct Cost-Reliability Problem}

Determine the optimal set of technical improvements $T I_{\xi_{q}}, 1 \leq q \leq Q \leq N, \xi_{q} \leq N$, which requires the minimal amount of costs to undertake the $T I$ in order to increase the device's reliability by not less than $R^{*}-R_{0}$, i.e.,

$$
\underset{\left\{\xi_{q}\right\}}{\operatorname{Min}}\left\{\sum_{q=1}^{Q} \Delta C_{\xi_{q}}\right\}
$$

subject to

$$
R_{0}+\sum_{q=1}^{Q} \Delta R_{\xi_{q}} \geq R^{*}
$$

\subsection{The Dual Cost-Reliability Problem}

Determine the optimal set of $T I_{\xi_{q}}, 1 \leq q \leq Q \leq N$, $\xi_{q} \leq N$, in order to maximize the device's reliability subject to the restricted amount of costs $\Delta C$ to undertake the corresponding $T I$, i. e.,

$$
\underset{\left\{\xi_{q}\right\}}{M a x}\left\{\sum_{q=1}^{Q} \Delta R_{\xi_{q}}\right\}
$$

subject to

$$
\sum_{q=1}^{Q} \Delta C_{\xi_{q}} \leq \Delta C
$$

Since all $T I$ are independent of each other, both problems (4-5) and (6-7) are NP- complete, and, an optimal solution can be obtained only by means of implementing an algorithm (mainly by means of dynamic programming) that checks the feasibility of all possible combinations of $Q$ elements from $N$, while $Q$ itself changes from 1 to $N$. If $N$ is high enough, the corresponding algorithm requires a lot of computational time according to the justification outlined in [1]. We suggest using a heuristic procedure based on cost-sensitivity. Note, that if relation

$$
\sum_{i=1}^{Q} \Delta R_{i} \geq R^{*}-R_{0}
$$

does not hold, the direct problem (4-5) has no solution. As to the dual problem, the corresponding restriction

$$
\sum_{i=1}^{Q} \Delta C_{i} \leq C^{*}
$$

results in a trivial solution $Q=N$, i.e., all technical investments have to be implemented.

\subsection{The Direct Problem's Solution (Algorithm I)}

In order to proceed, we will introduce a new definition. Call henceforth the cost-reliability of a technical improvement the ratio $\gamma=\Delta R / \Delta C$. It can be well-recognized that if $T I_{k_{1}}$ has a higher costreliability than $T I_{k}$, investing one and the same cost expenditure results in a higher increase of the reliability parameter in case of implementing the $T I_{k_{1}}$ than $T I_{k_{2}}$. This consideration is used below, in the step-by-step heuristic algorithm:

Step I Calculate cost-reliability values $\gamma_{k}$ for all $T I_{k}, 1 \leq k \leq N$.

Step 2 Reorder values $\gamma_{k}$ in descending order. Thus, values $\gamma_{k}, 1 \leq k \leq N$, will obtain a new order. Denote the corresponding new indices (ordinal numbers) of technical improvements by $T I_{\xi_{q}}, 1 \leq q \leq N$.

Step 3 Determine the minimal value $Q$ which satisfies

$Q=\operatorname{Min}\left[V: \sum_{q=1}^{V} \Delta R_{\xi_{q}} \geq R^{*}-R_{0}\right]$.

Step 4 Determine the quasi-optimal indices of the chosen technical improvements:

$$
T I_{\xi_{1}}, T I_{\xi_{2}}, \ldots, T I_{\xi_{Q}} \text {. }
$$


The idea of Algorithm I is to spend as little budget as possible in order to meet constraint (5).

\subsection{The Dual Problem's Solution (Algorithm II)}

The corresponding step-by-step heuristic algorithm is as follows:

Steps 1 and 2 fully coincide with the corresponding stages of Algorithm I.

Step 3 Determine the maximal value $Q$ which satisfies

$$
Q=\operatorname{Max}\left[V: \sum_{q=1}^{V} \Delta C_{\xi_{q}} \leq C^{*}\right] .
$$

Step 4 fully coincides with Step 4 of Algorithm I.

It can be well-recognized that introducing the concept of cost-reliability enables a simple and effective solution of various cost-optimization problems in Safety Engineering.

Both optimization problems (4-5) and (6-7) are partial harmonization problems. Problem (4-5) is a $P H M_{1}(R)=C$ with one independent basic parameter - system's reliability value $R$, and one dependent parameter - the budget to be assigned for undertaking technical improvements. $P H M_{1}(R)=C$ centers on minimizing $C$ subject to the prescribed reliability. Problem (6-7) is a $\operatorname{PHM}_{2}(C)=R$ which centers on maximizing the reliability value $R$ subject to restricted budget value $C$.

\section{Harmonization models in safety engineering}

\subsection{Cost-Reliability Harmonization Model with Two Basic Independent Parameters}

We will consider an interesting case (and for certain multi-level technical systems an important one!) of a system with possible hazardous failures at the upper level. Two independent basic parameters are imbedded in the model: budget $C$ to carry out technical improvements, and the system's reliability value $R$. In order to simplify the problem assume that, similarly to the model outlined above, all technical improvements are additive, i.e., additional system's reliability $\Delta R\left\{\xi_{1}, \ldots, \xi_{Q}\right\}$ obtained by implementing

$$
\left\{T I_{\xi_{q}}\right\}, 1 \leq q \leq Q \text {, is equal } \sum_{q=1}^{Q} \Delta R_{\xi_{q}} .
$$

Set the "weight" of increasing the device's reliability (per reliability unit) by $\alpha_{r}$, and let the corresponding weight of cost investments per cost unit be $\alpha_{c}$. The harmonization model is an extension of the costreliability model outlined in Chapter 2. The problem is as follows: Determine the optimal set of $\left\{T \xi_{\xi_{q}}\right\}$, $1 \leq q \leq Q, \quad \xi_{q} \leq N$, in order to maximize the harmonization objective

$$
J=\underset{\left\{\xi_{q}\right\}}{\operatorname{ax}}\left\{\sum_{q=1}^{Q}\left[\alpha_{r} \cdot \Delta R_{\xi_{q}}-\alpha_{c} \cdot \Delta C_{\xi_{q}}\right]\right\}
$$

subject to

$$
\sum_{q=1}^{Q} \Delta C_{\xi_{q}} \leq C^{*}
$$

$$
\sum_{q=1}^{Q} \Delta R_{\xi_{q}} \geq R^{*}-R_{0}=\Delta R
$$

Note that since the costs $\Delta C_{\xi_{q}}$ to be invested in the course of undertaking $T I \xi_{q}$ decrease the system's utility, i.e., decrease objective (12), they have to be taken with a negative sign, while increasing the device's reliability results in increasing the quality of the system as a whole.

Model (12-14) comprises two restrictions since for both basic parameters $C$ and $R$ their corresponding upper and lower bounds are pre-given. It is a complicated NP-complete problem which requires only heuristic solutions, since using classical precise optimization algorithms meets unavoidable computational difficulties. We suggest to solve problem (12-14) by implementing the idea of costsensitivity, based on introducing values $\gamma_{k}=\frac{\Delta R_{k}}{\Delta C_{k}}$, $1 \leq k \leq N$, and, later on, reordering $T I_{k}, 1 \leq k \leq N$, in the descending order of values $\gamma_{k}$. Thus, sequence $T I \xi_{q}, 1 \leq q \leq N$, is obtained.

To develop a heuristic procedure, we will modify objective (12) as follows:

$$
\begin{aligned}
& J=\underset{\left\{\xi_{q}\right\}}{\operatorname{ax}}\left\{\sum_{q=1}^{Q}\left[\left(\frac{\alpha_{r} \cdot \Delta R_{\xi_{q}}}{\alpha_{c} \cdot \Delta C_{\xi_{q}}}-1\right) \cdot \alpha_{c} \cdot \Delta C_{\xi_{q}}\right]\right\}= \\
& =M_{\left\{\xi_{q}\right\}} \operatorname{ax}\left\{\sum_{q=1}^{Q}\left[\left(\eta \cdot \gamma_{\xi_{q}}-1\right) \cdot \alpha_{c} \cdot \Delta C_{\xi_{q}}\right]\right\},
\end{aligned}
$$

where $\eta=\frac{\alpha_{r}}{\alpha}$ is a constant value which does not depend on the $T I$ index. 
Since values $\gamma_{\xi_{q}}$ are monotonously decreasing, the multiplicand $\eta \cdot \gamma_{\xi_{q}}-1,1 \leq q \leq N$, may for a certain number $q$ turn negative.

Certain realistic assumptions are imbedded in the model:

1. Since the reliability parameter for a technical device with critical failures usually dominates over other parameters, we will assume that relation

$$
\eta=\frac{\alpha_{r}}{\alpha_{c}}>1 \text { holds. }
$$

2. Assume that for the $T I_{\xi_{1}}$ with the highest costsensitivity, relation $\eta \cdot \gamma_{\xi_{1}}>1$ holds, otherwise a degenerate conclusion can be drawn that the best compromise for the device under consideration is to not undertake any technical improvements at all.

On the basis of the above assumptions the following step-by-step heuristic algorithm to solve harmonization problem (12-14) can be suggested:

Step 1. Determine the maximal $N_{1}$ satisfying

$$
N_{1}=\operatorname{Max}\left[V: \sum_{q=1}^{V} \Delta C_{\xi_{q}} \leq C^{*}\right] .
$$

Step 2. Determine the minimal $N_{2}$ satisfying

$$
N_{2}=\operatorname{Min}\left[V: \sum_{q=1}^{V} \Delta R_{\xi_{q}} \geq \Delta R\right] \text {. }
$$

Note that if $N_{2}>N_{1}$, the problem has no solution. In case $N_{2} \leq N_{1}$ apply Step 3 .

Step 3. Determine the maximal $N_{3}$ satisfying

$$
N_{3}=\operatorname{Max}\left[V: \eta \cdot \gamma_{\xi_{V}} \geq 1\right\rfloor
$$

subject to

$$
N_{3} \leq N .
$$

Step 4. Determine value $Q$ satisfying

$$
Q=\left\{\begin{array}{llr}
N_{1} & \text { if } & N_{3} \geq N_{1} \\
N_{3} & \text { if } & N_{2} \leq N_{3} \leq N_{1} . \\
N_{2} & \text { if } & N_{3} \leq N_{2}
\end{array}\right.
$$

Step 5. Technical improvements $T I_{\xi_{1}}, T I_{\xi_{2}}, \ldots, T I_{\xi_{Q}}$ are taken as the quasi-optimal set $\{T I\}$ to be implemented, with objective

$$
J=\sum_{q=1}^{Q}\left[\left(\eta \cdot \gamma_{\xi_{q}}-1\right) \cdot \alpha_{c} \cdot \Delta C_{\xi_{q}}\right] .
$$

Objective (21) honors restrictions (13-14) and delivers the maximal value for the problem's heuristic solution $\{T I\}_{\xi_{q}}, \quad 1 \leq q \leq Q$.

Note, in conclusion, that the ratio $\eta=\frac{\alpha_{r}}{\alpha_{c}}$ may not be a constant value. In case of extremely high reliability values $R$, i.e., when $R$ practically guarantees avoiding hazardous failures and relation $R \gg R^{*}$ holds, the partial utility value $\alpha_{R}$ may undergo an essential decrease while value $\alpha_{C}$ will remain constant. Thus, certain technical difficulties may arise. However, from the principal point of view, the algorithm will not be subject to drastic changes.

In the harmonization model under consideration a straightforward heuristic method [14] to optimize objective (12) is used. As to partial harmonization models, they do not exist in this case, since there are no dependent basic parameters: both basic parameters are set by means of restrictions (13-14) and are pregiven beforehand. No parameter is optimized by means of partial harmonization. Both parameters influence one another: this mutual influence is implemented in the heuristic algorithm by means of analyzing partial utility values $\alpha_{C}$ and $\alpha_{R}$.

\section{Generalized harmonization models in safety engineering with non-basic parameters}

The harmonization model under consideration comprises, besides two basic parameters $C$ (the budget to be assigned to undertake technical improvements) and $R$ (the system's reliability to avoid hazardous failures), a variety of non-basic parameters entering the system's utility model as well. Non-basic parameters are, e.g., the probability of completing the production program not later than the pre-given due date, reliability value to avoid nonhazardous failures which nevertheless may cause certain damage to the personnel and/or to the environment, specific design failures, etc. Unlike the outlined above cost-reliability models, all $T I$ are nonadditive, i.e., the aggregate increase $\Delta R\left\{\xi_{1}, \ldots, \xi_{Q}\right\}$ due to simultaneous implementation of $T I_{\xi_{1}}, \ldots, T I_{\xi_{Q}}$, may not be equal $\sum_{q=1}^{Q} \Delta R_{\xi_{q}}$. This makes the harmonization problem more complicated.

Two problems can be formulated: 


\subsection{Direct Problem}

Determine $Q \leq N$ technical improvements $T \xi_{\xi_{q}}$, $1 \leq q \leq Q, \quad \xi_{q} \leq N$, to maximize the system's additional utility

$$
\begin{aligned}
& \underset{\left\{\xi_{q}\right\}}{\operatorname{ax}}\left[\sum_{q=1}^{Q} \Delta U_{s \xi_{q}}\right]= \\
& =\operatorname{M}_{\left\{\xi_{q}\right\}} a_{q}\left\{\sum_{q=1}^{Q}\left[\alpha_{C} \cdot \Delta C_{\xi_{q}}+\left(\sum_{\ell=1}^{m} \alpha_{P_{\ell}} \cdot \Delta P_{\ell \xi_{q}}\right)\right]+\alpha_{R} \cdot \Delta R\left(\xi_{1}, \xi_{2}, \ldots, \xi_{Q}\right)\right\}
\end{aligned}
$$

subject to

$$
R_{0}+\Delta R\left(\xi_{1}, \xi_{2}, \ldots, \xi_{Q}\right) \geq R^{*}
$$

\subsection{Dual Problem}

$$
\underset{\left\{\xi_{q}\right\}}{\operatorname{ax}}\left[\sum_{q=1}^{Q} \Delta U_{s \xi_{q}}\right]
$$

subject to

$$
\sum_{q=1}^{Q} \Delta C_{\xi_{q}} \leq C^{*}
$$

It can be well-recognized that solving both problems (22-23) and (24-25) by means of precise algorithms results in tremendous and practically unavoidable computational difficulties. We suggest developing enhanced heuristic procedures based on sensitivity analysis. Two basic sensitivity values for each $T I_{k}$ will be used:

reliability-sensitivity $\omega_{k}=\frac{\Delta U_{s k}}{\Delta R_{k}}$,

and cost-sensitivity $\eta_{k}=\frac{\Delta U_{s k}}{\Delta C_{k}}, 1 \leq k \leq N$.

Note that both values $\omega_{k}$ and $\eta_{k}, 1 \leq k \leq N$, can be obtained only by means of simulation, since $\Delta U_{s k}$ comprises $\Delta R_{k}$ and has to be calculated via simulation with an enormous number of simulation runs.

\subsection{The Problem's Solution}

The enlarged step-by-step Algorithm I to solve the direct problem is as follows:
Step 1. Calculate reliability-sensitivity values $\omega_{k}$ for all $T I_{k}, 1 \leq k \leq N$.

Step 2. Reorder values $\omega_{k}$ in descending order. Thus, values $\omega_{k}$ will obtain a new order. Denote the corresponding new indices (ordinal numbers) of technical improvements by $T I_{\xi_{q}}, 1 \leq q \leq N$.

Step 3. Determine the minimal value $Q$ satisfying

$$
Q=\operatorname{Min}\left[V: \Delta R\left(\xi_{1}, \ldots, \xi_{V}\right) \geq R^{*}-R_{0}\right] .
$$

Step 4. If $\Delta U_{S Q}<0$ go to the next step. Otherwise go to Step 6.

Step 5. If value $Q$ exceeds the minimal value obtained at Step 3 go to Step 8. Otherwise go to Step 9.

Step 6. If $Q=N$ go to Step 9. Otherwise apply the next step.

Step 7. Counter $Q+1 \Rightarrow Q$ works. Go to Step 4 .

Step 8. $Q-1 \Rightarrow Q$. Apply the next step.

Step 9. Determine the quasi-optimal indices of the chosen technical improvements to be implemented:

$$
T I_{\xi_{1}}, T I_{\xi_{2}}, \ldots, T I_{\xi_{Q}}
$$

The step-by-step procedure of Algorithm II to solve the dual problem is as follows:

Step 1. Calculate cost-sensitivity values $\eta_{k}$ for all $T I_{k}, 1 \leq k \leq N$.

Step 2. Reorder values $\eta_{k}$ in descending order, similarly to Step 2 of Algorithm I.

Step 3. Determine the maximal value $Q$ satisfying

$$
Q=\operatorname{Max}\left[V: \sum_{q=1}^{Q} \Delta C_{\xi_{q}} \leq C^{*}\right] .
$$

Step 4. If $\Delta U_{S Q} \leq 0$ go to the next step. Otherwise apply Step 7.

Step 5. If $Q=1$ go to Step 7. Otherwise apply the next step.

Step 6. Counter $Q-1 \Rightarrow Q$ works. Go to Step 4 . 
Step 7. Determine the quasi-optimal solution of Algorithm II, i.e., the quasi-optimal sub-set of $\left\{T I \xi_{q}\right\}, 1 \leq q \leq Q$.

Algorithms I and II cover a broad spectrum of Safety Engineering problems.

Note that the direct harmonization problem (22-23) is based on $(m+1)$ partial harmonization models with $R$ being an independent parameter: $P_{H} H M_{1}(R)=C$, $\operatorname{PHM}_{1 \ell}(R)=P_{\ell}, 1 \leq \ell \leq m$, which later on enter the utility increment $\Delta U_{s}$. As to the dual problem (24$25)$, it comprises another $(m+1) P H M$ with budget value $C$ being an independent parameter:

$$
\operatorname{PHM}_{2}(C)=R, \operatorname{PHM}_{2 \ell}(C)=P_{\ell}, 1 \leq \ell \leq m .
$$

In conclusion, partial utility parameters $\alpha_{R}$ and $\alpha_{\ell}$, $1 \leq \ell \leq m$, in practice, are usually piecewise functions depending on the parameters' values. This causes certain computational difficulties in solving harmonization problems. However, those difficulties do not inflict principal troubles and can be overcome.

\section{Conclusions}

The following conclusions can be drawn from the study:

1. Problems of estimating the utility of complicated and usually multilevel management systems by means of establishing and solving harmonization problems are very urgent, especially for organization systems with a variety of quality parameters. Applications of the utility theory in recent publications are restricted to market competitive models and do not deal as yet with complicated hierarchical systems' functioning. The nowadays existing multi-attribute utility theory can be applied only to the stage preceding the product's design and determining the objectives for future market competition.

2. We suggest implementing the utility concept as a generalized system's quality estimate which takes into account several essential parameters. The latter usually define the quality of the system as a whole. We have developed a generalized harmonization problem in order to maximize the system's utility. The corresponding model is optimized by means of a two-level heuristic algorithm. At the upper level (the level of independent parameters) a relatively simple search procedure, e.g. the cyclic coordinate algorithm, has to be implemented. At the lower level partial harmonization problems to optimize the dependant parameters, have to be used. Note, that nowadays there is no formalized linkage between the system's parameters and attributes and, thus, no optimization problem can be put and solved in order to maximize the product's utility within its specific life cycle. The developed research enables implementing such a linkage, in future, on the stages of both designing and creating new products and, later on, on the stage of marketing the product.

3. Harmonization approaches in Reliability and Safety Engineering have been successfully used to develop various cost-reliability optimization models. The latter are applicable to a broad spectrum of hierarchical technical systems with a possibility of hazardous failure at the top level and a pre-given multi-linkage of failure elements at different levels. Such systems cannot be analyzed by means of former publications $[5,6,12]$.

4. In order to obtain quasi-optimal solutions of harmonization problems in Reliability and Safety Engineering, we have implemented the sensitivity analysis in the corresponding optimization algorithms. Sensitivity values (e.g. cost-reliability sensitivity) have been successfully utilized for developing heuristic computational techniques.

\section{Acknowledgement}

This research has been partially supported by the Paul Ivanier Center on Robotics and Production Management, Ben-Gurion University of the Negev.

\section{References}

1. Ben-Yair, A. (2004). Harmonization models in strategic management and safety engineering, Ph.D. Thesis, BenGurion University of the Negev, Beer-Sheva, Israel.

2. Ben-Yair, A. and Laslo, Z. (2004). Upon analyzing and synthesizing multi-level industrial systems with hazardous failures, Communications in Dependability and Quality Management, 7(2), 36-45.

3. Ben-Yair, A., Menipaz, E. and Papic, L. (2001). Heuristic cost-reliability models for non-compound devices in safety engineering, Communications in Dependability and Quality Management, 4 (2), 88-93.

4. Burkov, V.V. and Irikov, V.A. (1994). Models and Methods for Controlling Organization Systems, Moscow: Nauka (in Russian).

5. Kuo, W. and Prasad, V.P. (2000). An annotated overview 
of system - reliability optimization, IEEE Transactions on Reliability, R-49, 176-187.

6. Li, D. and Haimes, Y.Y. (1992). A decomposition method for optimization of large system reliability, IEEE Transactions on Reliability, R-41, 183-188.

7. Menipaz, E. (1984). Essentials of Production and Operations Management, Engle- wood Cliffs, N.J.: Prentice Hall, New York.

8. Menipaz, E. and Ben-Yair, A. (2001). Optimal harmonization model in multi- parametrical organization systems, Communications in Dependability and Quality Management, 4 (2), 12-24.

9. Papic, L., Ben-Yair, A. and Sinuany-Stern, Z. (2000). Upon reducing top critical failure probabilities by costoptimization models, Communications in Dependability and Quality Management, 3 (1), 47-56.

10. Papic, L., Ben-Yair, A. and Menipaz, E. (2003). Practical applications of harmonization models in hierarchical industrial enterprises, Communications in Dependability and Quality Management, 6 (1), 18-31.

11. Papic, L., Campean, F. and Khan, M. (2000). Safety concept in system effective- ness, Communications in Dependability and Quality Management, 3 (1), 6-10.

12. Sakawa, M. (1981). Optimal reliability - design of a series - parallel system by a large - scale multi-objective optimization method, IEEE Transactions on Reliability, R-30, 173-174.

13. Shtub, A., Bard, J. and Globerson, Sh. (1994). Project Management: Engineering, Technology and Implementation, Prentice Hall International, Inc., New York.

14. Taha, H.A. (1987). Operations Research: An Introduction, MacMillan, New York.

15. Wilpert, B. and Qvale, T. (eds.) (1993). Reliability and Safety in Hazardous Work Systems, Lawrence Erlbaum Associates, Hove (U.K.), Hillsdale (U.S.A.). 